\title{
BMJ Open Decision-making for people with dementia and advanced kidney disease: a secondary qualitative analysis of interviews from the Conservative Kidney Management Assessment of Practice Patterns Study
}

\author{
Jemima Scott, ${ }^{1,2}$ Amanda Owen-Smith, ${ }^{2}$ Sarah Tonkin-Crine, ${ }^{3}$ Hugh Rayner, ${ }^{4}$ \\ Paul Roderick, ${ }^{5}$ Ikumi Okamoto, ${ }^{5}$ Geraldine Leydon, ${ }^{5}$ Fergus Caskey, ${ }^{1,2,6}$ \\ Shona Methven ${ }^{6,7}$
}

To cite: Scott J, OwenSmith A, Tonkin-Crine S, et al. Decision-making for people with dementia and advanced kidney disease: a secondary qualitative analysis of interviews from the Conservative Kidney Management Assessment of Practice Patterns Study. BMJ Open 2018;8:e022385. doi:10.1136/ bmjopen-2018-022385

- Prepublication history and additional material for this paper are available online. To view these files, please visit the journal online (http://dx.doi. org/10.1136/bmjopen-2018022385).

Received 15 February 2018 Revised 26 July 2018 Accepted 28 September 2018

Check for updates

(c) Author(s) (or their employer(s)) 2018. Re-use permitted under CC BY-NC. No commercial re-use. See rights and permissions. Published by BMJ.

For numbered affiliations see end of article.

Correspondence to

Dr Jemima Scott;

jemimascott@doctors.org.uk

\section{ABSTRACT}

Objective To explore dialysis decision-making for adults who lack capacity due to cognitive impairment, a common and under-recognised condition in those with advanced chronic kidney disease (CKD).

Design Secondary analysis of qualitative data collected during the Conservative Kidney Management Assessment of Practice Patterns Study programme of research was performed. Sixty semistructured interviews were conducted with multiprofessional team members from UK renal centres. Staff were asked about local facilities, the value of conservative kidney management (CKM), when and with whom CKM was discussed and how CKM could be improved. Thematic analysis was employed to identify, characterise and report on themes that emerged from the data, focused on the specific issues experienced by people with dementia.

Setting A purposive sample of nine UK renal centres differing in the scale of their CKM programmes.

Participants Clinical directors of renal centres identified staff involved in CKM. Staff were asked to participate if they had experience of low clearance clinics or of caring for patients with advanced CKD (estimated glomerular filtration rate $<20 \mathrm{~mL} / \mathrm{min} / 1.73^{2}$ or $>65$ years with endstage kidney disease).

Results Two overarching themes were identified: factors taken into consideration during decision-making, and the process of decision-making itself. Comorbidity, social support, quality of life and the feasibility of dialysis were reported as factors pertinent to clinicians' decisions regarding suitability. The majority of renal centres practised multidisciplinary 'best interests' decision-making for those without capacity. Attitudes to advance care planning were divided.

Conclusions In view of the prevalence of cognitive impairment among those with advanced CKD, we suggest consideration of routine assessment of cognition and capacity. In the UK, dialysis is initiated and continued for individuals with dementia and services should be adapted to meet the needs of this population.
Strengths and limitations of this study

- This is the first qualitative analysis of this size to assess the opinions of renal healthcare professionals regarding the management of end-stage kidney disease for people with dementia.

- Purposive sampling of renal units and the breadth of professionals involved in these interviews provided granular data for study.

- This is a secondary analysis of data collected for an alternative purpose: lack of specific questions regarding patients with dementia limited the scope of investigation with respect to our specific research question.

\section{INTRODUCTION}

Dementia is characterised by a decline in one or more cognitive domains, severe enough to interfere with activities of daily living (ADLs). ${ }^{1}$ Mild cognitive impairment (MCI) may be a precursor to dementia, in which impaired cognition exists but is insufficient to effect independence or ADLs. ${ }^{2}$ A 2009 meta-analysis of Mayo-defined MCI showed progression to dementia in $32.3 \%$ (95\% CI $17.9 \%$ to $48.5 \%$ ) over a mean 4.6 years (SD 2.14). ${ }^{3}$

Chronic kidney disease (CKD) is an independent risk factor for cognitive impairment. ${ }^{4}$ Its prevalence in those with CKD is widely under-recognised. ${ }^{5}{ }^{6} \mathrm{~A}$ prospective cohort study found $61 \%$ of those with CKD4-5 had a Mini-Mental State Exam (MMSE) score $\leq 24$ at baseline, indicative of significant impairment. ${ }^{7}$ Orientation-attention and language skills are predominantly affected which may specifically impact on an individual's ability to 
engage with healthcare decisions. ${ }^{8}$ In a qualitative study of patients with Alzheimer's disease and their carers, patient involvement in medical decision-making declined significantly once the MMSE score fell below $20 .^{9}$ The UK Renal Association recommends that 'most patients whose eGFR is $<30 \mathrm{~mL} / \mathrm{min} / 1.73 \mathrm{~m}^{2}$ and declining should receive timely and personalised information regarding established kidney failure and renal replacement therapy options so they can make an informed decision about treatment'. ${ }^{10}$ Given the complexity of decision-making regarding renal replacement therapy (RRT), those with cognitive impairment struggle without support and some will lack capacity.

There are multiple treatment options for end-stage kidney disease (ESKD): haemodialysis (in a healthcare setting or at home), peritoneal dialysis (at home, performed by the patient or an informal carer) including assisted automated peritoneal dialysis (assisted APD) (supported by daily staff visits) or conservative kidney management (CKM) (supportive treatment and symptom control). There is a growing appreciation of CKM as an alternative to dialysis for older patients with multimorbidity and poor prognosis, ${ }^{11-13}$ but a lack of consensus on how to identify this group. In a recent survey of UK renal centres, all but one reported having an 'alternative to dialysis' pathway; however, the percentage of patients over 75 years managed on this pathway varied from less than $10 \%$ to $40 \%-50 \%$. $^{14}$ Renal transplantation is rarely appropriate for people with dementia due to the progressive nature of the disorder and associated comorbidity. ESKD decision-making is clinically challenging, emotive and preference sensitive. Relative benefits and disadvantages of treatment options differ between patients as variation in physical health and patients' values ${ }^{1516}$ influence the trade-off between quality versus quantity of life. Guidelines based purely on clinical trial data risk conflict with patient-centred care unless preference sensitivity is accounted for. ${ }^{17}$

In the UK, USA and Europe, four legal standards must be met to demonstrate capacitous decision-making: (1) to understand information given regarding the proposed treatment and expected risks and benefits, (2) to retain this information for sufficient time to reach a decision, (3) to be able to weigh up the advantages and disadvantages and (4) to communicate a decision. ${ }^{18-20}$ Several models of decision-making have been described for those lacking capacity: advance decisions, substituted judgement and best interests. Advance care plans (ACP) enable the individual to refuse future treatment, although not to demand care. ${ }^{21}$ Substituted judgement attempts to replicate the choice an individual would have made, had he been capacitous. This is frequently used in the USA. ${ }^{22}$ In the UK, the majority of such decisions use the 'best interests' of the patient, as set out in the Mental Capacity Act 2005. In this model, surrogate decision-makers, carers and professionals choose what they believe to be the best action at the current time and in the future. ${ }^{22}$

The doctor-patient relationship has evolved and there is now an expectation that the patient should make, or at least have significant input into, medical decisions. ${ }^{23} 24$ However, as dementia progresses, the degree of input the individual has into any given decision diminishes and, over time, ${ }^{9}$ many lose capacity to make competent decisions regarding health and medical care. The degree of autonomy is dependent on the question being asked as well as individual characteristics of patient and clinician. Jayanti et al demonstrated that older patients were less likely to want active involvement in decision-making. ${ }^{25}$ Other predictors of low engagement included high comorbidity score, poor executive brain function and lack of functional independence. ${ }^{25}$

This study contributes to our understanding of how decisions regarding dialysis versus CKM are made for patients with cognitive impairment who lack capacity.

\section{METHODS \\ Design and setting}

This study reports a secondary analysis of qualitative data collected by the Conservative Kidney Management Assessment of Practice Patterns Study (CKMAPPS) programme of research (see online supplementary appendix 1), conducted in England 2011-2013. CKMAPPS was designed to determine current UK practice patterns for CKM and to inform service development and future research. The programme comprised three qualitative and one quantitative study. Purposive sampling was used to identify nine renal centres across the UK differing in the scale of their CKM programmes. Within these centres, semistructured interviews were conducted with members of the renal multiprofessional team (see 'Participants' for details of sampling strategy). A favourable ethical opinion was obtained from South Birmingham National Research Ethics Committee (11/WM/0240). The original analysis is reported elsewhere. ${ }^{14}$

\section{Sampling}

Clinical directors of renal centres identified staff involved in CKM. Staff were asked to participate if they had experience of low clearance clinics or of caring for patients with advanced CKD (estimated glomerular filtration rate $<20 \mathrm{~mL} / \mathrm{min} / 1.73^{2}$ or $>65$ years with ESKD). A minimum of one consultant nephrologist and one staff nurse were recruited from each unit.

\section{Interviews}

Prior written consent was obtained from all participants. Semistructured interviews were conducted face to face or by telephone. There was no pre-existing relationship between the participants and the interviewers, who were experienced qualitative researchers from the University of Southampton. The interview schedule (see online supplementary appendix 2) was designed using a prior literature review and input from steering committee members, and adapted using an iterative process as participants introduced new topics. Staff were asked about how CKM was delivered, the role of primary and palliative care services, 
their views on CKM and experiences of involvement in CKM decision-making. Interviews were audio-recorded digitally and transcribed verbatim.

\section{Data analysis}

A secondary thematic analysis was conducted to identify themes within interviews with relevance to decision-making for adults who lack capacity as a result of cognitive impairment. NVivo V.10 qualitative data analysis software (QSR International) was used to facilitate coding. Each transcript was read by one of two clinical researchers, JS and SM, who were not involved in the original data collection process. Codes attached to the text highlighted significance with regards to the study question. Eight interviews were coded independently by both researchers to check consistency in the identification of relevant themes. Differences were resolved by discussion. Consensus was reached on an initial coding framework and applied to the remainder of the transcripts (divided between the two researchers). Saturation of themes related to cognitive impairment was achieved within this secondary analysis; however, this was not an explicit objective of data collection.

The initial framework was further developed through an iterative process as data analysis continued. Individual codes were built into a thematic framework, which was used to structure the analysis reported in this article. Particular attention was paid where there were differences in views expressed between interviewees, which were used as a platform for further in-depth analysis.

\section{Patient involvement}

The original CKMAPPS programme of the study was planned in collaboration with Kidney Alliance, a body that aims to bring together the patients' voice and professionals committed to renal medicine. The Chair of Kidney Alliance was part of the research team. Through this organisation, patients were involved in informing the study design and outcomes, as well as drafting patient information resources. Patients were not directly involved in recruitment. The results of the original CKMAPPS study were shared with patients via the Chair of Kidney Alliance.

\section{RESULTS}

Sixty staff interviews were undertaken in the initial CKMAPPS study (six to nine per unit). Participants included 22 nephrologists, 25 nurses, one palliative care consultant and 12 allied health professionals (six social workers, three dieticians, two counsellors, one pharmacist). Forty-seven participants were women. Median age was 49 years (range 28-67). Forty-seven participants described their ethnicity as White-British, five as WhiteOther, three as Asian British-Indian, two as Asian-Indian, one Asian-Other Asian, one Asian, one Mixed White and Asian and one as Fijian.

Fifty-nine interviews were included in this secondary analysis (one interview with a renal information technology systems manager was not transcribed). Two overarching themes were identified: factors taken into consideration during decision-making, and the process of decision-making itself. Several minor themes emerged within each core concept and are numbered below, with illustrative quotations.

\section{FACTORS TAKEN INTO CONSIDERATION DURING DIALYSIS DECISION-MAKING \\ Comorbidity and prognosis}

CKM was most frequently considered for individuals with multimorbidity, especially for those with dementia, cardiac disease or malignancy, due to lack of symptomatic and survival benefit.

For patients who are over 75 , who have got lots of illnesses... dialysis isn't guaranteed to make them live longer or feel better....those patients who have got a number of co-morbidities, so patients who have got heart failure, COPD, dementia... conservative management is very, very appropriate.(Specialist Nurse, Unit 8)

However, one nurse commented that the patient's psychological state was more important than their physical health with regards to the tolerability and success of dialysis.

I think a lot of elderly [person] people ...they have a zest for life and however physically immobile they are, they still do incredibly well because it's about your psyche and your capacity to cope with dialysis as opposed to your physical ability to cope with it. (Staff Nurse, Unit 5)

Some staff suggested that cognitive impairment might negatively impact on the success of RRT, while dialysis itself might accelerate mental decline.

I suppose the classic thing [in the CKM clinic] is there are often very significant vascular [paths], amputations, heart attacks, strokes-and they sometimes have a significant element of cognitive declineand I know that that may well get worse on dialysis. (Consultant Nephrologist, Unit 8)

\section{The feasibility of dialysis}

Clinicians commented on the feasibility of dialysis. Physical and psychological health problems were seen as potential barriers to successful RRT. Some clinicians advocated a trial of dialysis, with cessation of treatment if the patient exhibited distress or the staff or patient were put at risk by the patient's behaviour.

It's not only [difficult] for them [patients with dementia]; it's difficult to look after them. To dialyse them is pretty hard.' (Consultant Nephrologist, Unit 1) 
Despite this, several participants reported that it was not uncommon for patients with dementia to be started on dialysis; good outcomes were noted for some.

Some of the patients who don't have capacity perhaps, who we have best interest meetings and then the decision is for the patient actually to have dialysis, and it...has worked really well. (Social Worker, Unit 3)

Assisted APD and CKM were proposed as alternative options for those unable to tolerate haemodialysis. Staff suggested the extra support received with assisted APD could overcome the challenge of ensuring a patient was physically and cognitively able to manage the technique.

We've got our own assisted APD programme as well so that's helped quite a lot with patients that perhaps can't cope with dialysis themselves or can't cope with coming in and out for [haemo] dialysis. (Staff Nurse, Unit 3)

\section{Quality of life}

Many staff reported taking into account a patient's quality of life (QoL), specifically considering cognitive impairment, when considering suitability for RRT. Clinicians recognised deterioration in the QoL in patients receiving haemodialysis; many felt this was reason to avoid dialysis in those with poor QoL at baseline.

I've got an old lady um, who lives in the nursing home, her husband has got dementia, she has got an element of dementia too, and she is having four hours of dialysis every other day. and she is bed bound, she is stretchered in, stretchered out, hoisted... what quality of life have we? (Staff Nurse, Unit 6)

The increased time spent in hospital as well as travelling to and from dialysis was believed to be particularly detrimental to QoL.

Sometimes the [more] frailer people, if they are on dialysis, end up having more hospital admissions: just things like the burden of travelling backwards and forwards to the dialysis unit. (Consultant Nephrologist, Unit 7)

\section{The provision of services for ESKD}

Clinicians suggested that where there was a lack of assisted APD, frail patients were more likely to receive CKM.

Those patients [receiving assisted APD] would have [otherwise] been for non-dialysis care in many senses because haemodialysis would have been too distressful. (Matron, Unit 3)

While underprovision in one modality was felt to discourage referrals, clinicians felt that good local services actively encouraged referral for that pathway.

Because we have got an active conservative management programme my colleagues feel you know although the patient is elderly we can still offer them something. ...You feel that you can do something proactive. (Consultant Nephrologist, Unit 1)

However, a nurse expressed concerns that patients may feel pressured into accepting a specific dialysis modality solely because of availability.

Because there is an option to use assisted APD, there's a chance and a risk that it would be used inappropriately...to just attach someone to a machine because the opportunity is there, I don't feel is appropriate. (PD Nurse, Unit 4)

\section{Clinicians' perceptions of social support}

Good family support was seen by many as key to the success of dialysis for those with dementia, but also important in the delivery of good quality CKM. Clinicians expressed uncertainty as to which modality was preferable in those without such support networks.

It is more difficult to keep people well on dialysis treatment if they have poor social support but it is also more difficult to maintain a good quality of life for them with conservative management. (Consultant Nephrologist, Unit 5)

Some nursing staff felt that family support was of greater importance to dialysis success than age or clinical condition. Doctors were more likely to mention clinical factors and longevity.

We offer dialysis to everybody....just because they've got a learning disability or a little bit of dementia, if they are pleasantly confused and coping and good family support, they can have it. (Education Nurse, Unit 9)

Staff recognised the potential for both dialysis and CKM to offer social support to patients and their families. The CKM specialist nurse was described as a pivotal link between medical and social services.

If they'd (CKM patients) like a bit more help, we can get the district nurses to do an assessment... you can...get the Macmillan nurses involved. (CKM Specialist Nurse, Unit 6)

In addition, dialysis was suggested to provide respite for carers.

I've had one or two cases where they [carers] don't want them [patients] at home, they would rather pack them off for haemodialysis... It just gives them the opportunity to have a bit of a rest. (Staff Nurse, Unit 6)

\section{PROCESSES INVOLVED IN DIALYSIS DECISION-MAKING} Ethico-legal aspects of decision-making

Participants discussed ethical and legal implications of making dialysis decisions for patients lacking capacity. 
Some suggested that the complexity of information relating to RRT may limit the numbers of patients able to make capacitous decisions regarding the management of ESKD.

For some patients, particularly the elderly, who are really starting to experience vascular problems-and have an element of vascular cognitive impairment, just understanding anything about renal failure or anything about what's happening to them, is so complicated. (Consultant Nephrologist, Unit 8)

The legal framework of best interests decision-making was used in cases where it was deemed that the patient lacked capacity.

We will make the decision on their behalf [for patients with severe cognitive impairment] and then we will say patient's best interest or medical decision. (Consultant Nephrologist, Unit 1)

Participants from five units reported being involved in regular multidisciplinary best interests meetings, involving clinicians, social workers and often the general practitioner, while others did not.

We don't have a discussion forum where we...or a multidisciplinary meeting where we discuss patients with these decisions [r/e RRT]. (Consultant Nephrologist, Unit 5)

In these centres, the individual consultant appeared to have the greatest influence on choice of dialysis modality.

Ultimately it [dialysis decision] comes down to the Consultant, really, that's looking after the patient. (Staff Nurse, Unit 2)

The majority of staff preferred family members to be in agreement with the decision made.

If a person has got some degree of dementia [they would be appropriate for CKM] ....we'd always try to involve the family....not just the doctors we'd try and involve the family agreeing with that decision [for CKM]. (Staff Nurse, Unit 3)

Staff commonly believed that families tended to wish for aggressive treatment of their relative, due to a lack of appreciation of the rigours of dialysis, unrealistic expectations and unwillingness to accept mortality.

I get patients with mobility problems and dementia problems and vascular problems and their relatives....believe that dialysis will cure all of the above. (Consultant Nephrologist, Unit 8)

One consultant commented on the Lasting Power of Attorney, and the challenge of ensuring that they are truly acting in the patient's best interests.

What is very difficult is to have a patient without capacity, somebody with Power of Attorney for their health needs and that person making decisions that are not, in our view, in the interests of the patient. (Consultant Nephrologist, Unit 7)

\section{The role of advance care planning}

There was significant disparity between both units and individual clinicians regarding the benefits and disadvantages of ACPs. Some argued that ACPs avoided the need to make hasty decisions and afforded adequate time to prepare the patient both physically and psychologically for the chosen treatment modality. ACPs were described as particularly advantageous in the context of declining cognition.

You can head that situation off [best interests decision making] by having advanced discussions when the patient has full capacity. (Consultant Nephrologist, Unit 7).

Others believed early decision-making was inappropriate. Reasons given included avoiding unnecessary distress should the patient never reach ESKD, the inability of patients to anticipate how they may feel in the future and the possibility of a change in health status such that an earlier decision is rendered inappropriate.

One [patient] had a little bit of dementia but she was very, very pleasant and was great coming to clinic ... she was all ready ... [but] round the time she started dialysis - she had just got a little bit more confused.... didn't do well, didn't want it, was very confused, kept wanting to pull to pull her dialysis needles out, screamed-you know-just didn' t cope. (Education Nurse, Unit 9)

\section{DISCUSSION}

In this secondary analysis of CKMAPPS, interviews with renal centre staff were analysed to understand the decision-making processes regarding ESKD management for patients with cognitive impairment who lack capacity. Participants reported using the framework of best interests decision-making and were divided as to their appreciation of ACPs. In the initial CKMAPPS survey of 67 UK renal units, $87.9 \%$ held regular multidisciplinary team meetings for difficult RRT decisions; however, in these interviews, staff from several centres suggested that the primary decision-maker was the individual consultant. Key influences on these decisions included comorbidity, the feasibility of dialysis and the clinician's perception of the patient's QoL and social care. Individual consultant preference and the local provision of services also impacted on the outcome. CKM was generally thought to be the most appropriate option for patients with cognitive impairment who lack capacity. Nevertheless, several reports emerged of such patients being commenced on dialysis.

The UK Mental Capacity Act 2005 states that a Best Interests Meeting involves 'the considered input of a 
number of different staff as well as those with a personal and/or legal interest in the needs of the person lacking mental capacity.... (and takes) into consideration all relevant circumstances, including the person's beliefs and values, past and present wishes, and any written statements the person made when she/he had capacity'. ${ }^{26}$ Participants in this study were generally unwilling to make best interests decisions regarding dialysis without the family's agreement; however, some expressed doubt regarding the ability of relatives to act as reliable surrogate decision-makers. Similar concerns have been raised previously. ${ }^{27-30}$ Markes and Arkes ${ }^{31}$ found that next of kin based decisions on what they themselves would have wanted, rather than reflecting the patient's wishes. Discordance widened between patient and surrogate decision-maker where the patient's preference was to refuse treatment. ${ }^{32}$ In this study, some clinicians believed ACPs might overcome these difficulties, although those in opposition suggested early RRT discussions might cause unnecessary distress for individuals who would never reach ESKD. ${ }^{33}$ Such concerns are challenged by a study of patients recently diagnosed with dementia, which showed overwhelmingly positive results from ACPs for both patient and caregiver. ${ }^{34} \mathrm{ACP}$ is a core component of the Department of Health for England's End of Life Care Strategy, ${ }^{35}$ and has been shown to increase QoL, reduce hospitalisations and increase home deaths. ${ }^{36}$ ACPs may be of particular benefit to those with progressive cognitive impairment where deterioration in decision-making capability may be anticipated. Despite this, less than $20 \%$ of patients with dementia are believed to have an ACP in the UK. ${ }^{37}$

This analysis raises issues of relevance to current clinical practice. First, given the prevalence of cognitive impairment in those with advanced CKD, the introduction of regular assessments of cognition and capacity into routine clinical practice should be considered. Screening tools such as the Montreal Cognitive Assessment and the Mini-Addenbrookes Cognitive Examination have been validated for both dialysis and CKD patients. ${ }^{38}{ }^{39}$ Identification of early cognitive impairment may prompt clinicians to initiate ACPs before loss of decision-making capability. ACPs should be regularly revisited to ensure that treatment preferences remain appropriate in the presence of changing comorbidity, cognitive function and QoL.

Second, there is a pressing need for the development of ESKD services (CKM, peritoneal and haemodialysis) which meet the needs of those with dementia. Given the rise in average age at dialysis initiation in the UK, the clustering of cardiovascular risk factors leading to ESKD and dementia and the growing burden of comorbidity for those living to ever greater ages, the coexistence of dementia in those receiving dialysis will almost certainly rise. This is in contrast to the overall plateau in dementia prevalence seen in the UK in recent years. The additional staff training, costs and time required to develop such programmes may be difficult to achieve in the face of increasing financial pressures within healthcare.

This study is the first qualitative analysis of this scale to assess the opinions of renal healthcare professionals regarding ESKD management for patients with cognitive impairment, a growing concern in the ageing population. Purposive sampling of renal centres and the breadth of professionals involved in these interviews should allow transferability of results to other UK renal centres. Despite this, limitations are recognised. This secondary analysis of interview data was collected for an alternative purpose, assessing attitudes to delivery of CKM. Lack of specific questions regarding patients with cognitive impairment will limit the potential richness of the data collected with regards to our specific research question.

Further research is required to determine the acceptability of screening for cognitive impairment in the advanced CKD population, the frequency of screening required and whether formal diagnosis changes management. Thought should also be given to potential disadvantages of diagnosis. It is important to investigate the attitudes of those with cognitive impairment to ACP with regard to RRT, whether attitudes change with disease progression and if care plans hold weight when individuals reach ESKD. Understanding the impact of different ESKD management modalities on QoL of those with cognitive impairment will influence future service development.

\section{CONCLUSIONS}

This secondary analysis of the CKMAPPS advances our understanding of dialysis decision-making for patients with cognitive impairment who lack capacity. Comorbidity, social support, QoL and the feasibility of dialysis were reported as factors pertinent to clinicians' decisions regarding suitability. The majority of renal centres practised multidisciplinary team 'best interests' decision-making and were unwilling to act against the wishes of relatives. Attitudes to ACP, which may offer particular benefit to those with progressive cognitive impairment, were divided. We suggest that in view of the prevalence of cognitive impairment among those with advanced CKD, the benefits and disadvantages of routine assessment of cognition and capacity for these individuals should be determined and consideration made to integration of such assessments into routine clinical practice. In the UK, dialysis is both initiated and continued for individuals with dementia and services should be adapted to meet the needs of this population.

\section{Author affiliations}

${ }^{1}$ Richard Bright Renal Unit, Southmead Hospital, Bristol, UK

${ }^{2}$ Department of Social \& Community Medicine, University of Bristol, Bristol, UK

${ }^{3}$ Nuffield Department of Primary Care Health Sciences, University of Oxford, Bristol, UK

${ }^{4}$ Department of Renal Medicine, Heart of England NHS Foundation Trust, Birmingham, UK 
${ }^{5}$ Primary Care \& Population Sciences, Faculty of Medicine, University of Southampton, Southampton, UK

${ }^{6}$ UK Renal Registry, Bristol, UK

${ }^{7}$ Department of Renal Medicine, Aberdeen Royal Infirmary, Aberdeen, UK

Acknowledgements The authors are grateful to members of the Kidney Alliance who provided valuable feedback on the design of the original CKMAPPS study. They would also like to thank the anonymous reviewers who reviewed an earlier version of this work and whose comments helped the authors to make improvements to the paper.

Contributors All authors meet the ICMJE conditions for authorship. The original CKMAPPS programme of work was designed by FC, PR, HR and GL and the interviews undertaken by ST-C and IO. SM designed this secondary analysis. JS and SM performed the data analysis and drafting of the manuscript with assistance from experienced qualitative researcher AO-S. JS, SM, AO-S, ST-C, I0, FC, PR, HR and GL contributed to data analysis and revision of the manuscript and approved the final draft.

Funding The authors have not declared a specific grant for this research from any funding agency in the public, commercial or not-for-profit sectors.

Competing interests Publication costs are covered by an NIHR Academic Clinical Fellowship awarded to JS

Patient consent Not required.

Ethics approval South Birmingham National Research Ethics Committee.

Provenance and peer review Not commissioned; externally peer reviewed.

Data sharing statement All requests should be directed to the corresponding author.

Open access This is an open access article distributed in accordance with the Creative Commons Attribution Non Commercial (CC BY-NC 4.0) license, which permits others to distribute, remix, adapt, build upon this work non-commercially, and license their derivative works on different terms, provided the original work is properly cited, appropriate credit is given, any changes made indicated, and the use is non-commercial. See: http://creativecommons.org/licenses/by-nc/4.0/

\section{REFERENCES}

1. Petersen RC, Association AP. Diagnostic and statistical manual of mental disorders. 5th edn, 2013. (DSM-5).

2. Petersen RC. Mild cognitive impairment as a diagnostic entity. $J$ Intern Med 2004;256:183-94.

3. Mitchell AJ, Shiri-Feshki M. Rate of progression of mild cognitive impairment to dementia-meta-analysis of 41 robust inception cohort studies. Acta Psychiatr Scand 2009;119:252-65.

4. Etgen $\mathrm{T}$, Chonchol M, Förstl $\mathrm{H}$, et al. Chronic kidney disease and cognitive impairment: a systematic review and meta-analysis. $\mathrm{Am} \mathrm{J}$ Nephrol 2012;35:474-82.

5. Murray AM, Tupper DE, Knopman DS, et al. Cognitive impairment in hemodialysis patients is common. Neurology 2006;67:216-23.

6. Nulsen RS, Yaqoob MM, Mahon A, et al. Prevalence of cognitive impairment in patients attending pre-dialysis clinic. J Ren Care 2008;34:121-6.

7. Foster R, Walker S, Brar R, et al. Cognitive impairment in advanced chronic kidney disease: the canadian frailty observation and interventions trial. Am J Nephrol 2016;44:473-80.

8. Berger I, Wu S, Masson P, et al. Cognition in chronic kidney disease: a systematic review and meta-analysis. BMC Med 2016;14:206.

9. Hirschman KB, Xie SX, Feudtner C, et al. How does an Alzheimer's disease patient's role in medical decision making change over time? J Geriatr Psychiatry Neurol 2004;17:55-60.

10. Farrington K, Warwick G. Renal Association Clinical Practice Guideline on planning, initiating and withdrawal of renal replacement therapy. Nephron Clin Pract 2011;118:c189-208.

11. Murtagh FE, Marsh JE, Donohoe P, et al. Dialysis or not? A comparative survival study of patients over 75 years with chronic kidney disease stage 5. Nephrol Dial Transplant 2007;22:1955-62.

12. Morton RL, Snelling P, Webster AC, et al. Factors influencing patient choice of dialysis versus conservative care to treat end-stage kidney disease. CMAJ 2012;184:E277-E283.

13. Chandna SM, Da Silva-Gane M, Marshall C, et al. Survival of elderly patients with stage 5 CKD: comparison of conservative management and renal replacement therapy. Nephrol Dial Transplant 2011;26:1608-14.
14. Roderick P, Rayner H, Tonkin-Crine S, et al. Health services and delivery research. A national study of practice patterns in UK renal units in the use of dialysis and conservative kidney management to treat people aged 75 years and over with chronic kidney failure. Southampton (UK): NIHR Journals Library.

15. Puhan MA, Yu T, Stegeman I, et al. Benefit-harm analysis and charts for individualized and preference-sensitive prevention: example of low dose aspirin for primary prevention of cardiovascular disease and cancer. BMC Med 2015;13:250.

16. Ubel PA. Medical Facts versus Value Judgments-Toward PreferenceSensitive Guidelines. N Engl J Med 2015;372:2475-7.

17. Keirns CC, Goold SD. Patient-centered care and preference-sensitive decision making. JAMA 2009;302:1805-6.

18. Karel MJ, Gurrera RJ, Hicken B, et al. Reasoning in the capacity to make medical decisions: the consideration of values. J Clin Ethics 2010;21:58-71.

19. Appelbaum PS, Grisso T. Assessing patients' capacities to consent to treatment. N Engl J Med 1988;319:1635-8.

20. Tepper AM, Elwork A. Competence to consent to treatment as a psychological construct. Law Hum Behav 1984;8:205-23.

21. Mental Capacity Act 2005. Nephrol dial transplant: Issued by the Lord Chancellor, 2007.

22. Joyce T, Interests B. Guidance on determining the best interests of adults who lack the capacity to make a decision (or decisions) for themselves [England and Wales]. 2008 bps.org.uk/system/files/ Public\%20files/Policy/rep_67_best_interests_web.pdf

23. Spatz ES, Krumholz HM, Moulton BW. The New Era of Informed Consent: Getting to a Reasonable-Patient Standard Through Shared Decision Making. JAMA 2016;315:2063-4.

24. NHS. Letter to doctors from the chief medical officer for scotland. 2015 http://www.gov.scot/Resource/0049/00492520.pdf.

25. Jayanti A, Neuvonen M, Wearden A, et al. BASIC-HHD study group. Healthcare decision-making in end stage renal diseasepatient preferences and clinical correlates. BMC Nephrol 2015;16:189.

26. Adults and Children Mental Capacity Act 2005. Best interests meetings guidance. 2012 http://www.devon.gov.uk/best_interest_ meetings guidance v8 july 2012.pdf (Accessed Jul 2018).

27. Shalowitz DI, Garrett-Mayer E, Wendler D. The accuracy of surrogate decision makers: a systematic review. Arch Intern Med 2006;166:493-7.

28. Kurella Tamura M, Montez-Rath ME, Hall YN, et al. Advance directives and end-of-life care among nursing home residents receiving maintenance dialysis. Clin J Am Soc Nephrol 2017;12:435-42

29. Fried TR, Gillick MR. The limits of proxy decision making: overtreatment. Camb Q Healthc Ethics 1995;4:524-9.

30. Tam-Tham H, Hemmelgarn BR, Campbell DJ, et al. Primary care physicians' perceived barriers, facilitators and strategies to enhance conservative care for older adults with chronic kidney disease: a qualitative descriptive study. Nephrol Dial Transplant 2016;31:1864-70.

31. Marks MA, Arkes HR. Patient and surrogate disagreement in end-of-life decisions: can surrogates accurately predict patients' preferences? Med Decis Making 2008;28:524-31.

32. Layde PM, Beam CA, Broste SK, et al. Surrogates' predictions of seriously ill patients' resuscitation preferences. Arch Fam Med 1995;4:518-23

33. Bansal N, Katz R, De Boer IH, et al. Development and validation of a model to predict 5-year risk of death without ESRD among older adults with CKD. Clin J Am Soc Nephrol 2015;10:363-71.

34. Poppe M, Burleigh S, Banerjee S. Qualitative evaluation of advanced care planning in early dementia (ACP-ED). PLoS One 2013;8:e60412.

35. DH. End of Life Care Strategy. 2008 https://www.gov.uk/government/ uploads/system/uploads/attachment_data/file/136431/End_of_life_ strategy.pdf (Accessed 17 Mar 2017).

36. Brinkman-Stoppelenburg A, Rietjens JA, van der Heide A. The effects of advance care planning on end-of-life care: a systematic review. Palliat Med 2014;28:1000-25.

37. Fetherstonhaugh D, McAuliffe L, Bauer M, et al. Decision-making on behalf of people living with dementia: how do surrogate decisionmakers decide? J Med Ethics 2017;43:35-40.

38. Hobson P, Rohoma KH, Wong SP, et al. The utility of the miniaddenbrooke's cognitive examination as a screen for cognitive impairment in elderly patients with chronic kidney disease and diabetes. Dement Geriatr Cogn Dis Extra 2016;6:541-8.

39. Paraizo MA, Almeida AL, Pires LA, et al. Montreal Cognitive Assessment (MoCA) screening mild cognitive impairment in patients with chronic kidney disease (CKD) pre-dialysis. J Bras Nefrol 2016;38:31-41. 